

\title{
Multiduoplasmatron et multiduopigatron : sources de plasma uniforme pour la formation de faisceaux d'ions multiamperes
}

\author{
C. Lejeune, J.P. Grandchamp, J. Aubert
}

\section{- To cite this version:}

C. Lejeune, J.P. Grandchamp, J. Aubert. Multiduoplasmatron et multiduopigatron: sources de plasma uniforme pour la formation de faisceaux d'ions multiamperes. Revue de Physique Appliquée, 1977, 12 (12), pp.1835-1848. 10.1051/rphysap:0197700120120183500 . jpa-00244411

HAL Id: jpa-00244411

https://hal.science/jpa-00244411

Submitted on 1 Jan 1977

HAL is a multi-disciplinary open access archive for the deposit and dissemination of scientific research documents, whether they are published or not. The documents may come from teaching and research institutions in France or abroad, or from public or private research centers.
L'archive ouverte pluridisciplinaire HAL, est destinée au dépôt et à la diffusion de documents scientifiques de niveau recherche, publiés ou non, émanant des établissements d'enseignement et de recherche français ou étrangers, des laboratoires publics ou privés. 


\title{
MULTIDUOPLASMATRON ET MULTIDUOPIGATRON : SOURCES DE PLASMA UNIFORME POUR LA FORMATION DE FAISCEAUX D'IONS MULTIAMPERES ${ }^{*}$ )
}

\author{
C. LEJEUNE, J. P. GRANDCHAMP et J. AUBERT \\ Institut d'Electronique Fondamentale, Laboratoire Associé au C. N. R. S. \\ Université Paris XI, Bâtiment 220, 91405 Orsay Cedex, France
}

(Reçu le 16 juin 1977, accepté le 16 septembre 1977)

\begin{abstract}
Résumé. - La formation de faisceaux d'ions intenses et peu divergents requiert pour le plasma la présence d'une surface émissive large, dense, quasi-uniforme et stable. Nous proposons deux structures de source capables de satisfaire à ces critères : le plasma est créé par plusieurs décharges planes et parallèles dont les courants sont indépendamment réglables. Pour la première structure - le Multiduoplasmatron - les décharges sont du type duoplasmatron; l'allumage des sept décharges disponibles permet de créer, dans une chambre cylindrique de $155 \mathrm{~mm}$ de diamètre, un plasma dont la surface émissive est uniforme à $\pm 3 \%$ sur un cercle de diamètre $120 \mathrm{~mm}$; en régime continu, et pour une puissance de décharge de $8 \mathrm{~kW}$, la densité du courant d'ions positifs, au niveau de la surface émissive, atteint $40 \mathrm{~mA} . \mathrm{cm}^{-2}$ pour l'hydrogène et $13 \mathrm{~mA} . \mathrm{cm}^{-2}$ pour l'argon ; le rendement énergétique de production de ces ions vaut respectivement 2500 et $7000 \mathrm{eV}$. ion ${ }^{-1}$, et le rendement d'ionisation des neutres 30 et $60 \%$.

Pour la seconde structure - le Multiduopigatron - les décharges sont du type duopigatron, ce qui entraîne une forte amélioration du rendement en énergie de production des ions, d'où en régime continu, une augmentation du flux d'ions disponible. Les conditions d'adaptation des paramètres géométriques et de la topographie du champ magnétique pour optimiser le Multiduopigatron sont rapportées, pour une opération avec l'hydrogène ou l'argon, éléments dont les masses correspondent aux deux applications principales des faisceaux intenses; quatre décharges de même intensité suffisent alors pour obtenir dans la même chambre cylindrique une surface émissive uniforme à $\pm 2 \%$ sur un cercle de diamètre $120 \mathrm{~mm}$, le champ magnétique résiduel étant inférieur à $50 \mathrm{G}$; en régime continu, la densité du courant d'ions positifs, au niveau de la surface émissive, atteint $200 \mathrm{~mA} \cdot \mathrm{cm}^{-2}$ pour une puissance de décharge de l'ordre de $4 \mathrm{~kW}$; le rendement énergétique de production de ces ions est alors de $275 \mathrm{eV}$.ion ${ }^{-1}$, et le rendement d'ionisation des neutres vaut $30 \%$ pour l'hydrogène et $60 \%$ pour l'argon.
\end{abstract}

Abstract. - Formation of intense ion beams with low divergence requires the creation of a plasma with a large, dense, quiescient and quasi-uniform emissive boundary. We propose two source structures which satisfy these requirements. The plasma is created by several plane and parallel discharges, the currents of which are independly chosen. For the first structure - the Multiduoplasmatron - the discharges are of the duoplasmatron type ; the ignition of the seven available discharges permits to create within a $155 \mathrm{~mm}$ diameter cylindrical chamber, a plasma which emissive boundary is uniform to $\pm 3 \%$ over a $120 \mathrm{~mm}$ diameter section. For a $\mathrm{C}$. W. operation with a $8 \mathrm{~kW}$ discharge power, the current density of the positive ions impinging at the plasma boundary attains $40 \mathrm{~mA} . \mathrm{cm}^{-2}$ with hydrogen and $13 \mathrm{~mA} . \mathrm{cm}^{-2}$ with argon; the energy efficiency for the production of these ions is respectively 2500 and $7000 \mathrm{eV}$.ion-1 and the neutral utilization efficiency 30 and $60 \%$.

For the second structure - the Multiduopigatron - the discharges are of the duopigatron type which insures a large improvement of the ion production energy efficiency, and therefore an increase of the available ion flux for a C. W. operation. The conditions of the adaptation of both the geometric and magnetic parameters in order to optimize the Multiduopigatron for the operation either with hydrogen or argon are reported, the mass of these elements corresponding to the two main applications of intense ion beams. Four discharges having the same intensity are sufficient to create within the same cylindrical chamber, a plasma boundary uniformity to $\pm 2 \%$ over a $120 \mathrm{~mm}$ diameter section, the fringing magnetic field being smaller than $50 \mathrm{G}$. For the $\mathrm{C}$. W. operation, the current density of the positive ions impinging at the plasma boundary attains $200 \mathrm{~mA} . \mathrm{cm}^{-2}$ with a discharge power about $4 \mathrm{~kW}$; the energy efficiency for the production of these ions is then $275 \mathrm{eV}$. ion $^{-1}$ where as the neutral utilization efficiency is $30 \%$ for hydrogen and $60 \%$ for argon.

(*) Une communication sur ce sujet a été présentée au "Colloque Sources d'Ions " du Congrès National de Physique des Plasmas (Paris, Déc. 1976). Depuis, les performances ont été améliorées, en particulier en ce qui concerne l'opération avec l'hydrogène, par l'adaptation de la zone de compression des décharges. 
1. Introduction. - Des faisceaux intenses d'ions multiampères en continu sont désormais souhaités : des ions légers (hydrogène, hélium) pour l'injection dans les machines à fusion [1] et des ions lourds d'éléments gazeux ou mieux métalliques pour le traitement des surfaces [2]. Les considérations théoriques [3, 4], aussi bien que les observations expérimentales [5], montrent que la formation de faisceaux multiampères exploitables - en particulier peu divergents - tient à la possibilité de produire un plasma qui soit simultanément : étendu, dense, quasi-uniforme et stable, au niveau de la première électrode de formation du faisceau, celle qui détermine la position de la surface émissive, c'est-à-dire l'interface plasma-faisceau. Davis et al. [5] ont montré qu'en ce qui concerne la divergence du faisceau, l'uniformité est la considération la plus importante, et qui donc doit en premier lieu être optimisée. Le coût de l'installation (équipement de vide et de puissance...) impose la recherche du meilleur rendement énergétique de production des ions $\left(\mathrm{eV}\right.$.ion $\left.{ }^{-1}\right)$ et du meilleur rendement d'ionisation des neutres injectés. Un tel plasma idéal étant créé, la formation sous haute tension d'un faisceau multiampères en régime permanent pose des problèmes physiques [4] mais surtout technologiques [1] non encore résolus ; ils sont étudiés par différentes équipes engagées dans la réalisation d'un programme de fusion contrôlée [1].

En alternative aux structures de décharges mises en œuvre pour la réalisation de sources intenses [7], nous proposons, sur la base d'une expérimentation antérieure [8] une structure - le Multiplasmatron qui produit un plasma dont la surface émissive répond aux critères précédents. Ce plasma, en contact avec une électrode circulaire de diamètre $155 \mathrm{~mm}$, est créé par plusieurs décharges planes et parallèles allumées au sein d'une géométrie adéquate en présence d'un champ magnétique inhomogène ; les courants de ces décharges sont variables indépendamment et il est ainsi possible d'agir sur la répartition de densité ionique au niveau de la surface émissive. Les décharges peuvent être du type duoplasmatron et la structure est dite Multiduoplasmatron; ses performances sont présentées dans le $\S 2$. La mise en œuvre de l'effet reflex sur les électrons ionisants permet d'optimiser simultanément l'illumination de l'électrode de formation du faisceau et les rendements ; les conditions d'adaptation de la géométrie et du champ magnétique sont exposées dans le $\S 3$. Les décharges opèrent en duopigatron et la structure est du type Multiduopigatron. Différents types d'ions gazeux ont été testés. Cependant l'hydrogène et l'argon qui sont les éléments types correspondant aux applications des sources intenses, ont fait l'objet d'une étude plus approfondie en vue de la production d'ions positifs. En conclusion, nous situons les performances de ces structures par rapport à celles existantes et nous discutons les possibilités de leur adaptation en vue de la production de faisceaux intenses d'ions métalliques (Multiduopigatron) ou d'ions légers négatifs (Multiduoplasmatron).
2. Multiduoplasmatron. -2.1 DESCRIPTOIN DE LA SOURCE. - La figure 1 est une coupe schématique du multiduoplasmatron; il comprend sept chambres à décharge à double compression, géométrique et magnétique, du type duoplasmatron [9]. L'anode A et l'électrode intermédiaire E. I., communes aux sept décharges sont les pôles de la lentille magnétique de compression des plasmas; chacune des décharges a une cathode indépendante. Les fentes d'éjection des plasmas - au niveau de l'anode - sont régulièrement réparties dans un cercle de diamètre $155 \mathrm{~mm}$ correspondant à celui de la chambre - ou cuvette - d'expansion, qui est commune aux sept jets de plasma.

Un travail préalable sur maquette a permis de déterminer la forme à donner au circuit magnétique (en particulier aux pôles) en vue d'obtenir : 1) un champ de compression aussi identique que possible le long de chaque chambre et dans les différentes chambres et 2) un maximum de la composante longitudinale du champ au voisinage du plan des fentes d'anode. Le but

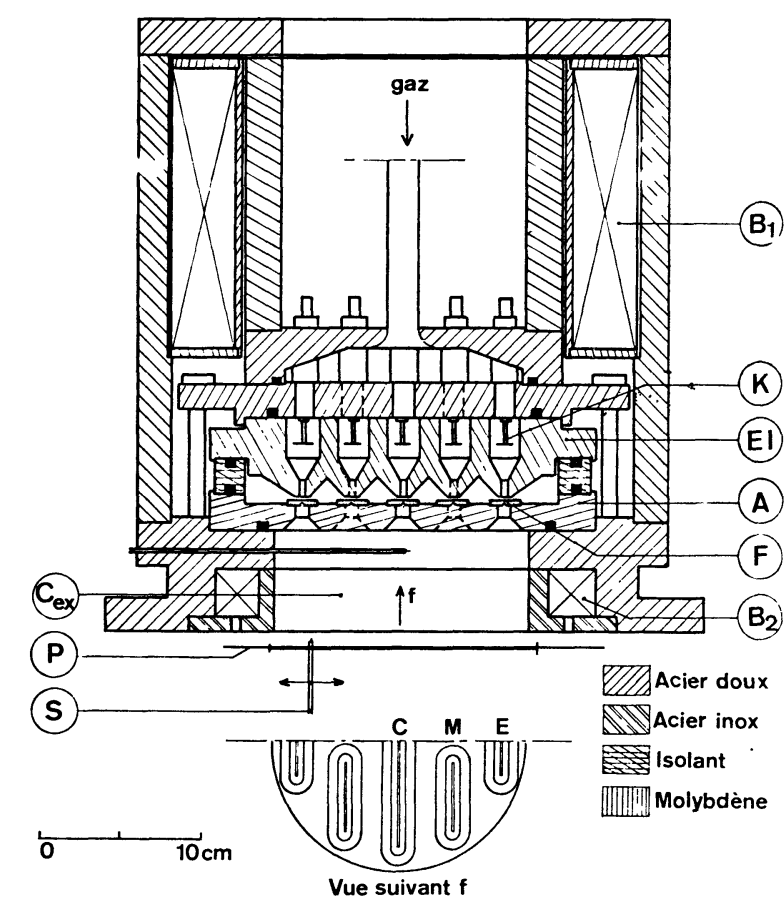

Fig. 1. - Multiduoplasmatron : Coupe selon le plan médian perpendiculaire aux plans des décharges $; \mathbf{B}_{1}$ : Bobine du champ magnétique de compression; $\mathrm{K}$ : Cathodes chauffées; $\mathrm{EI}$ : Electrode intermédiaire ; $\mathbf{A}:$ Anode ; $\mathbf{F}:$ Fentes d'éjection des plasmas; $\mathrm{B}_{2}$ : Bobine du champ magnétique d'expansion; $\mathrm{C}_{\mathrm{EX}}$ : Cuvette d'expansion; P : Plan du collecteur d'ions; $\mathrm{S}$ : Sonde électrostatique mobile. Les 7 décharges sont repérées par les qualificatifs extrêmes $(E)$, médianes $(M)$ et centrale $(C)$ : la vue suivant $f$ précise la répartition, dans le plan de l'anode, des fentes d'éjection des plasmas de ces décharges.

[Multiduoplasmatron : section through the source perpendicularly to the plane of the discharges; $\mathbf{B}_{1}$ : coil for the « compression magnetic field $» ; \mathrm{K}$ : heated cathodes ; EI : intermediate electrode ; A : anode ; F : plasma emission slits ; $\mathbf{B}_{2}$ : coil for the expansion magnetic field $; \mathrm{C}_{\mathrm{EX}}$ : expansion cup ; $\mathrm{P}:$ Position of the ion collector ; $\mathbf{S}:$ Electrostatic probe. The seven discharges are referred to as extreme (E), median (M) and central (C); the view $f$ from bottom shows the position in the anode of the seven emission slits.] 
a été atteint avec le circuit de la figure 1, pour des inductions allant jusqu'à $5 \mathrm{kG}$, valeur au-delà de laquelle la saturation se manifeste ; une seule bobine d'excitation $B_{1}$ suffit pour créer un tel champ. La figure 2

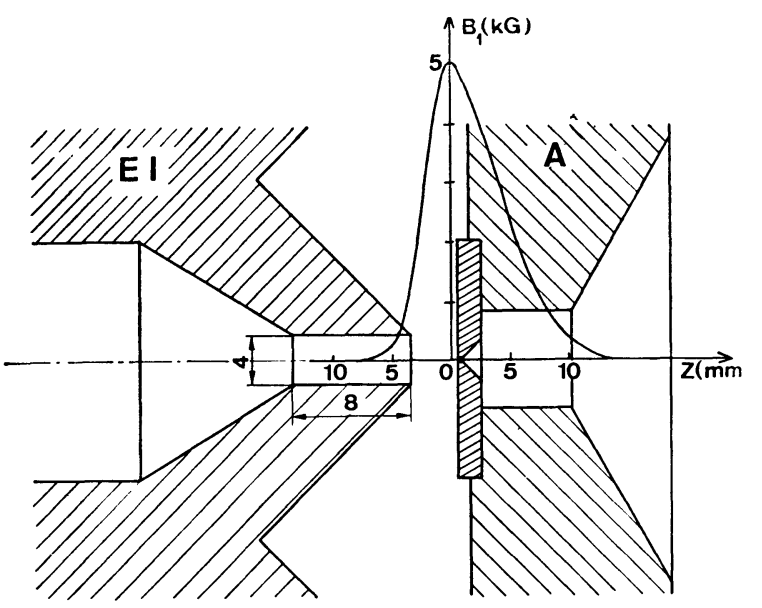

$$
\text { o } 5 \frac{1}{10}(\mathrm{~mm})
$$

Fig. 2. - La double compression - géométrique puis magnétique - d'une décharge duoplasmatron ; $B_{1}=f(z)$ est le profil de l'induction magnétique dans le plan de symétrie de la compression pour une intensité d'excitation de $4 \mathrm{~A}$ dans la bobine $\mathrm{B}_{1}$.

[The double - geometric and magnetic - compression of the duoplasmatron discharges ; $B_{1}=f(z)$ is the magnetic field profile within the symetry plane ( $B_{1}$ coil current $\left.4 A\right)$.]

donne en détail les dimensions transversales de la zone de compression géométrique et la variation de l'intensité du champ de compression dans le plan médian d'une décharge. Afin de contrôler l'effusion et la superposition des jets de plasma dans la cuvette d'expansion, une bobinc indépendante $B_{2}$ y crée un champ magnétique d'expansion de faible intensité (Fig. 3). La figure 4 est une photographie de la face d'éjection de l'anode, du nez de l'électrode intermédiaire et du pont magnétique supportant les cathodes : elle précise la forme des pôles magnétiques ainsi que la position des tuyaux assurant le refroidissement de ces électrodes.

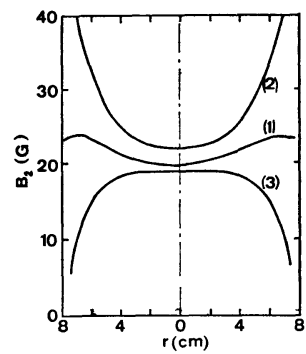

FIG. 3. - Variation, le long d'un diamètre de la cuvette d'expansion de la composante longitudinale de l'induction magnétique $B_{2}$ pour trois plans de section droite et une intensité magnétisante de $1 \mathrm{~A}$ dans la bobine $\mathbf{B}_{2}: 1$. Plan de sortie de la cuvette 2. Plan médian; 3. Plan voisin de l'anode.

[Variation of the longitudinal componant of the magnetic field $B_{2}$ along a cup diameter for three sections: 1. exit plane ; 2. median plane; 3 . plane close to the anode $\left(B_{2}\right.$ coil current $1 \mathrm{~A})$.]

Le refroidissement efficace ainsi obtenu permet, en régime permanent, de fonctionner jusqu'à une puissance de l'ordre de $8 \mathrm{~kW}$, pour l'ensemble des déchar-

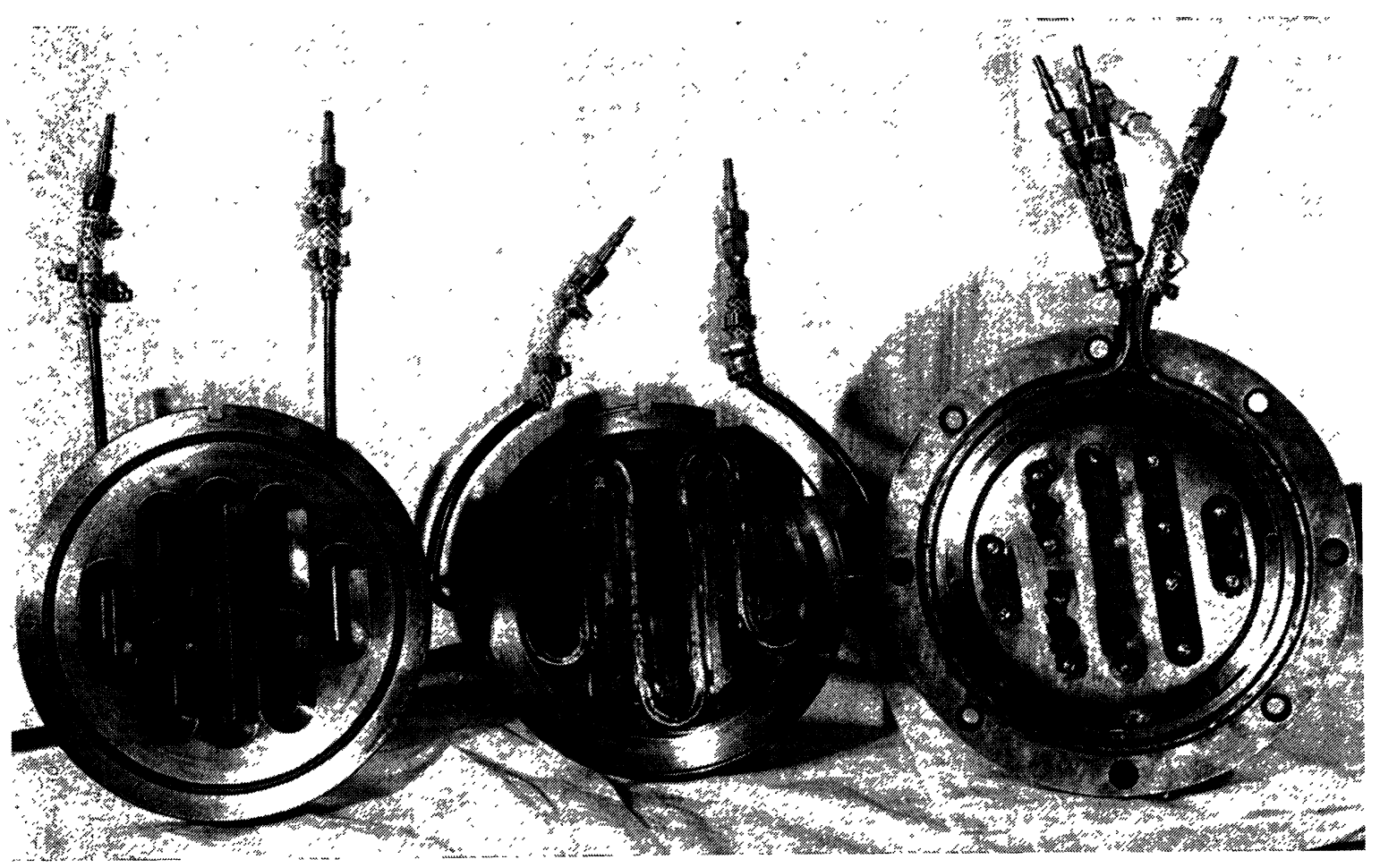

FIG. 4 - Multiduoplasmatron - de gauche à droite, photographie: de la face de l'anode (donnant sur la cuvette d'expansion); du nez de l'électrode intermédiaire, faisant face à l'anode ; du pont magnétique supportant les sept cathodes à oxydes.

[Multiduoplasmatron : from the left to the right, a photograph of : the anode, facint the expansion cup ; the intermediate electrode nose, facing the anode; the iron piece (magnetic bridge) which supports the seven oxyde cathodes.] 
ges, soit un courant total maximum de $80 \mathrm{~A}$ pour l'hydrogène et $120 \mathrm{~A}$ pour l'argon dans les conditions de pression qui maximisent le rendement d'ionisation (cf. $\S 2.2 .1$ ). Les cathodes - du type à oxydes sont réalisées au laboratoire; leur durée de vie, fonction du courant de décharge par suite de la pulvérisation ionique, est de l'ordre de 150 heures pour l'argon et 250 pour l'hydrogène. Compte tenu de l'uniformité recherchée et de la similitude des caractéristiques courant-tension des sept décharges, une seule source de puissance $150 \mathrm{~V}-120 \mathrm{~A}$ est parfaitement bien adaptée au problème. La modification des courants de décharge est alors obtenue soit par variation de la puissance de chauffage des cathodes (émission en température), soit par variation d'une résistance de charge en série dans le circuit de polarisation de chaque décharge, les cathodes surchauffées émettant en charge d'espace. Cette résistance détermine par ailleurs la stabilité du point de fonctionnement de chaque décharge. Ce montage assure la reproductibilité du point de fonctionnement global et permet, par variation de la tension de l'alimentation, de conserver la répartition des courants pour d'autres points de fonctionnement. L'électrode intermédiaire, reliée à l'anode par une faible résistance en vue de l'amorçage de la décharge, est ensuite laissée flottante.

Un système automatisé de sonde, mobile parallèlement ou perpendiculairement aux fentes d'anode, est mis en œuvre pour explorer les propriétés du plasma dans un plan de section droite de la cuvette d'expansion, choisi à priori; des sondes déplaçables sous chacune des fentes d'éjection complètent ce système. La majeure partie des mesures a été faite avec des sondes cylindriques : les anisotropies des fonctions de distribution des vitesses des charges ont été mises en évidence au moyen de sondes planes dont une seule face est collectrice et, de plus, orientable par rapport aux lignes de force du champ magnétique.

Comme déjà mentionné, notre objectif actuel n'est pas la formation d'un faisceau d'ions d'énergie donnée, mais la production d'un plasma émissif optimisé. Il se pose alors le problème de la caractérisation des performances de ce plasma et de la source d'ions qu'il serait possible de réaliser à partir de cette structure de décharge, en lui adaptant un système convenable d'extraction du type multiouvertures à trois électrodes :

- Le flux d'ions $I_{P}^{+}$disponible au niveau de la surface émissive du plasma d'une source à décharge ne dépend pas en première approximation de la tension d'accélération, entre quelques centaines de volts et quelques dizaines de $\mathrm{kV}$ [10]. C'est ce qui est observé ici lors de la collection, jusqu'à $2 \mathrm{kV}$, par une plaque $P$ placée en retrait du plan de sortie de la cuvette d'expansion (cf. Fig. 1) : la saturation $I_{\mathrm{P}}^{+}$est obtenue dès $200 \mathrm{~V}$, par rapport à l'anode. La transparence $t$ de l'optique d'extraction détermine alors le courant $I_{\mathrm{F}}^{+}$ du faisceau :

$$
I_{\mathrm{F}}^{+}=t I_{\mathrm{P}}^{+}
$$

- Le rendement d'utilisation du gaz injecté (débit $N_{0}$ molécules. $\mathrm{s}^{-1}$ ) se définit de la façon usuelle, c'est-àdire dans les ions monochargés correspondant aux molécules introduites par:

$$
\eta_{\mathrm{G}}=I_{\mathrm{P}}^{+} / e N_{0} \text {. }
$$

Il caractérise valablement la source, la transmission de l'optique affectant les flux de neutres et d'ions de facteurs très voisins (\# $t$ ).

- Le rendement énergétique de production des ions disponibles $\left(I_{\mathrm{P}}^{+}\right)$se définit par le rapport :

$$
\eta_{\mathrm{E}}=I_{\mathrm{D}} V_{\mathrm{D}} / I_{\mathrm{P}}^{+}, \quad\left(\mathrm{eV} . \text { ion }^{-1} \text { ou W. } \mathrm{A}^{-1}\right)
$$

$I_{\mathrm{D}}$ et $V_{\mathrm{D}}$ étant le courant total et la tension de la décharge. Avec extraction, il est à diviser par $t$.

- Les qualités (divergence, émittance) du faisceau formé à partir d'un tel plasma sont déterminées par les propriétés de sa surface émissive (uniformité, stabilité, fonction de distribution en énergie des ions et des électrons, champ magnétique résiduel) et celles de l'optique d'extraction.

- La surface émissive du plasma sera caractérisée par son profil de densité volumique de charges, ou mieux de densité de courant d'ions $j_{\mathrm{P}}^{+}$, grandeur qui fait intervenir la fonction de distribution de vitesse de ces ions.

2.2 RÉSUlTATS EXPÉRIMENTAUX. - 2.2.1 Les dé. charges "duoplasmatron linéaire ». - Les décharges s'allument uniformément sur toute leur longueur : les sondes le vérifient ; la densité du plasma est constante le long de chacune des décharges sur une distance égale à la longueur de sa fente d'éjection. Cette mesure est faite à $20 \mathrm{~mm}$ de ces fentes, c'est-à-dire avant que l'expansion du jet de plasma ne modifie sensiblement la répartition initiale de densité (cf. Fig. 6). Les caractéristiques courant-tension des sept décharges - étudiées séparément puis simultanément - présentent les propriétés intrinsèques du duoplasmatron. La tension $V_{\mathrm{D}}$ dépend principalement de la nature des neutres et de leur pression ; par accroissement de son courant, la décharge passe du régime normal au régime d'appauvrissement en neutres. Au changement de régime : la tension $V_{\mathrm{D}}$ correspond à l'énergie du maximum de la section efficace d'ionisation du gaz; le courant $I_{\mathrm{D}}$ est sensiblement proportionnel à la pression des neutres et à la longueur de la colonne ionisée ; le courant d'ions émis et le rendement d'ionisation passent par un maximum, d'autant plus grand que $I_{\mathrm{D}}$ est intense. L'extrapolation du modèle établi pour une décharge à symétrie cylindrique [9] interprète ces résultats.

2.2.2 Le plasma émissif. - Un large plateau de la densité de courant ionique peut être obtenu dans un plan de section droite de la cuvette, par le choix convenable de la répartition du courant entre les différentes décharges et de l'intensité $\left(B_{2}\right)$ du champ d'expansion. Le réglage de ces paramètres ne dépend, en première approximation, que de la nature des ions, l'intensité 
$\left(B_{1}\right)$ du champ de compression et du plan de section droite choisi. L'essentiel de notre étude a porté sur les profils de densité dans le plan de sortie de la cuvette d'expansion. Il présente en effet plusieurs avantages : le champ magnétique y est sensiblement uniforme (cf. Fig. 3) ; les profils partiels de densité dûs à chacune des décharges y sont suffisamment larges pour réaliser une superposition peu sensible aux variations dans la



FIG. 5. - Profils de densité de courant ionique $j_{\mathrm{p}}^{+}$le long d'un diamètre $\left(r_{\perp}\right)$ perpendiculaire aux décharges, dans le plan de sortie de la cuvette d'expansion. Hydrogène; débit : $2,1 \mathrm{~cm}^{3} \cdot \mathrm{s}^{-1}$ (T. P. N.) ; pression anodique $p_{\mathrm{A}} \simeq 2 \times 10^{-2}$ torr ; $V_{\mathrm{D}}=100 \mathrm{~V}$; courants de décharges, extrêmes : $I_{\mathrm{DE}}=2 \times 10 \mathrm{~A}$, médianes : $I_{\mathrm{DM}}=4 \times 7,5 \mathrm{~A}$; centrale $: I_{\mathrm{DC}}=10 \mathrm{~A}$. La comparaison des profils $b$ et $a$ montre l'effet de l'inversion du sens du champ magnétique d'expansion. Le profil « $c$ » montre, par comparaison à « $a$ », l'effet de l'extinction des décharges extrêmes.

[Transversal profiles of the ion current density $\left(j_{\mathrm{p}}^{+}\right)$along a diameter $\left(r_{\perp}\right)$ perpendicular to the discharge plane) at the cup exit plane. Hydrogen flow : $2.1 \mathrm{~cm}^{3} \cdot \mathrm{s}^{-1}$ (T. N. P.) ; anodic pressure : $p_{\mathrm{A}} \simeq 2 \times 10^{-2}$ torr ; $V_{\mathrm{D}}=100 \mathrm{~V}$; discharge currents ; extreme : $I_{\mathrm{DE}}=2 \times 10 \mathrm{~A}$; median : $I_{\mathrm{DM}}=4 \times 7.5 \mathrm{~A}$; central : $I_{\mathrm{DC}}=10 \mathrm{~A}$. The comparison of profiles $b$ and $a$ shows the effect of the inversion of the expansion magnetic field. The profile $c$ shows if compared to $a$, the effect of the extinction of the extreme discharges.]

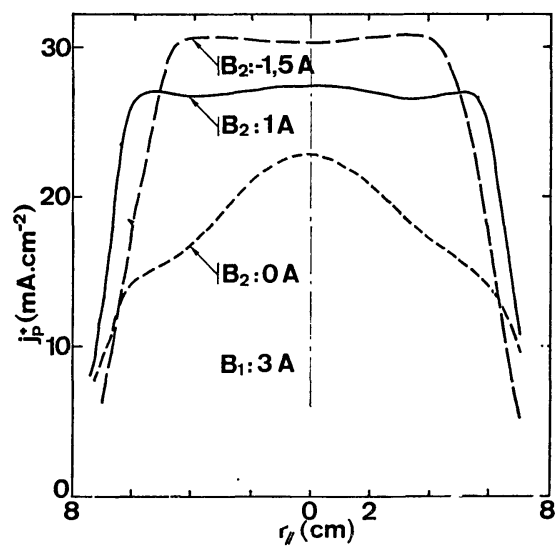

Fig. 6. - Profils de densité de courant ionique le long d'un diamètre $(r \bar{i})$ parallèle à la fente centrale pour trois valeurs du champ magnétique d'expansion $\mathbf{B}_{2}$. Les courants de décharge sont ceux de la figure 5 .

[Profiles of ion current density along a diameter $\left(r_{\|}\right)$parallel to the central slit ; the expansion magnetic field $B_{2}$ is the parameter ; the discharge currents are those given in the figure 5 caption.] distribution des courants; l'adaptation d'un système d'extraction à plusieurs électrodes n'y pose pas de problèmes particuliers de tenue en tension. Des exemples de profil de densité à large plateau sont donnés par les figures 5 et 6 , respectivement le long de deux diamètres perpendiculaire et parallèle aux décharges. $\mathrm{La}$ réduction de la dimension transversale du plateau, consécutive à l'extinction des deux décharges extrêmes, y est illustrée. (La distribution des courants des sept décharges et les paramètres sont donnés dans les légendes des figures). L'ensemble de notre étude conduit aux conclusions suivantes :

- Le plasma de la cuvette d'expansion, qui résulte de la superposition des jets issus des décharges, est un plasma sans collisions [11] dont la dynamique est dominée par les trajectoires individuelles de ses charges sous l'action du champ magnétique et des champs électriques de charge d'espace qui s'y développent. En effet, la pression y est très inférieure à celle régnant dans le compartiment anodique des décharges, et les libres parcours moyens de réactions sont très supérieurs aux dimensions de la cuvette. Il y a effusion et non diffusion des jets de plasmas. C'est pourquoi aussi les valeurs de $j_{\mathrm{p}}^{+}$portées en ordonnées des graphes sont calculées à partir du courant total collecté $I_{\mathrm{P}}^{+}$et de la surface d'émission, et non pas du courant recueilli par les sondes, dont l'exploitation ne relève pas du modèle simplifié usuel, par suite des anisotropies des vitesses des ions et des électrons. Les valeurs ainsi calculées sont cependant en bon accord avec celles mesurées à partir d'une sonde plane perpendiculaire au jet de plasma.

- Un champ magnétique d'expansion s'impose pour obtenir un plateau de densité. Il peut être soit de

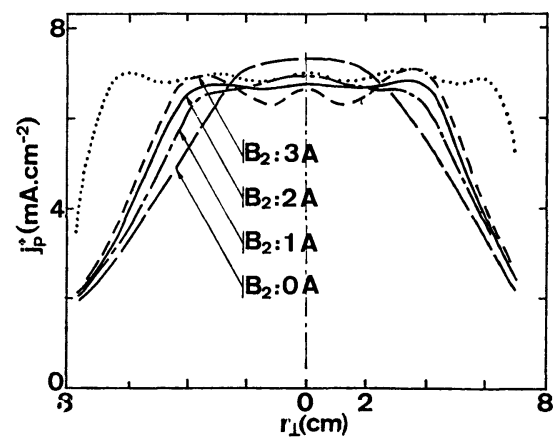

Fig. 7. - Influence du champ magnétique d'expansion sur le profil transversal de densité ionique, pour 5 décharge dans l'Argon : débit : $0,26 \mathrm{~cm}^{3} \cdot \mathrm{s}^{-1}$ (T. N. P.) $; p_{\mathrm{A}} \simeq 1,2 \times 10^{-2}$ torr ; $V_{\mathrm{D}}=45 \mathrm{~V} ; B_{1}: 4 \mathrm{~A} ; I_{\mathrm{DC}}=10 \mathrm{~A} ; I_{\mathrm{DM}}=4 \times 10 \mathrm{~A}$. En pointillés est figuré le profil obtenu par l'allumage des décharges extrêmes $\left(I_{\mathrm{DE}}=2 \times 11 \mathrm{~A}\right)$, pour le champ magnétique d'expansion optimal $\left(B_{2}^{*}: 2 \mathrm{~A}\right)$.

[Influence of the expansion magnetic field on the transversal profile of ion current density; five discharges with Argon; gas flow: $0.26 \mathrm{~cm}^{3} . \mathrm{s}^{-1}$ (T. P. N.) ; $p_{\mathrm{A}} \simeq 1.2 \times 10^{-2}$ torr ; $V_{\mathrm{D}}=45 \mathrm{~V} ; B_{1}=4 \mathrm{~A} ; I_{\mathrm{DC}}=10 \mathrm{~A} ; I_{\mathrm{DM}}=4 \times 10 \mathrm{~A}$. The dotted line is the observed profile when the extreme discharges are ignited $\left(I_{\mathrm{DE}}=2 \times 11 \mathrm{~A}\right)$, the magnetic field current being $B_{2}^{*}: 2$ A.] 
même sens, soit de sens contraire au champ de compression; le plateau de densité est cependant plus large pour une configuration à champs de même sens (Fig. 5 et 6). Pour chacune, le champ résiduel dans le plan de mesure est faible : entre 20 et $50 \mathrm{G}$ pour des inductions de compression variant de 3 à $6 \mathrm{kG}$ au niveau des fentes d'anode. La variation de $B_{2}$ au voisinage de la valeur optimale $B_{2}^{*}$ qui assure un plateau le plus large et le plus uniforme, n'entraîne pas de détérioration sensible de l'uniformité de densité de la surface émissive (Fig. 7, relative à l'argon); le réglage de $B_{2}$ aussi bien que celui de la distribution des courants des décharges n'est pas critique. Ces observations sont la conséquence de la largeur importante, en regard du diamètre de la cuvette, des contributions de chaque décharge (Fig. 8). A noter que la superposition des plasmas ne se fait pas de façon rigoureusement linéaire, par rapport à la sommation des densités partielles; la modification du champ électrique de charge d'espace explique ce résultat. Tant que la saturation du circuit magnétique n'est pas atteinte, $B_{2}^{*}$ est proportionnel à $B_{1}$ et la distribution des courants de décharge n'a pas à être modifiée ; la largeur du plateau de densité n'est pas sensiblement affectée, de telle sorte qu'en première approximation on a :

$$
B_{2}^{*} \sim B_{1} ; \quad I_{\mathrm{P}}^{+} \sim B_{1} \text { et } j_{\mathrm{P}}^{+} \sim B_{1} .
$$

Selon la masse des ions, les conditions de réglage

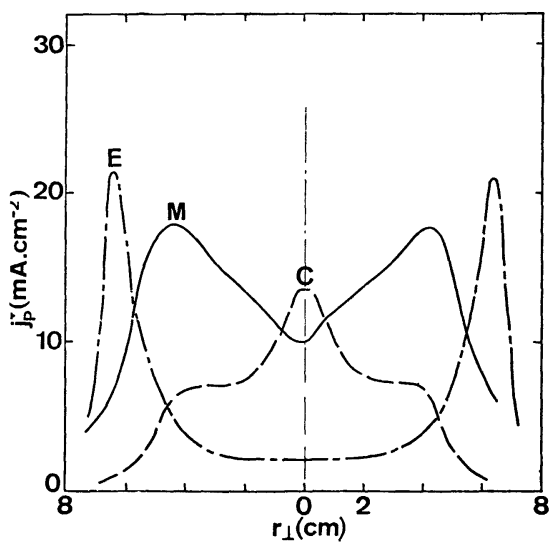

FIG. 8. - Profils partiels de densité correspondant respectivement aux décharges allumées symétriquement dans l'hydrogène. Décharges, centrale : $I_{\mathrm{DC}}=10 \mathrm{~A}$; médianes : $I_{\mathrm{DM}}=4 \times 10 \mathrm{~A}$; extrêmes : $I_{\mathrm{DE}}=2 \times 7,5 \mathrm{~A}$; pression : $2 \times 10^{-2}$ torr ; $V_{\mathrm{D}}=100 \mathrm{~V} ; B_{1}: 3 \mathrm{~A} ; B_{2}: 3 \mathrm{~A}$.

[Partial density profiles corresponding to the separate operation of the symetrical discharges : $I_{\mathrm{DC}}=10 \mathrm{~A} ; I_{\mathrm{DM}}=4 \times 10 \mathrm{~A}$; $I_{\mathrm{DE}}=2 \times 7.5 \mathrm{~A} ;$ hydrogen pressure $\simeq 2 \times 10^{-2}$ torr ; $\left.V_{\mathrm{D}}=100 \mathrm{~V} ; B_{1}: 3 \mathrm{~A} ; B_{2}: 3 \mathrm{~A}.\right]$

doivent être corrigées (cf. Fig. 5 pour $\mathrm{H}_{2}^{+}$et Fig. 7 pour $\mathrm{Ar}^{+}$) mais un plateau uniforme de même dimension est obtenu, $I_{\mathrm{P}}^{+}$et $j_{\mathrm{P}}^{+}$variant selon la loi en $M^{-1 / 2}$, usuelle pour le duoplasmatron [9].

- La configuration du champ magnétique étant

\section{Tableau I}

Performances optimales du Multiduoplasmatron ; le diamètre de la chambre d'expansion est $155 \mathrm{~mm}$ et la largeur des fentes d'éjection des jets de plasma $0,4 \mathrm{~mm}$; les dimensions de la zone de compression sont celles de la figure 2. La transparence de l'optique d'extraction affecte le flux d'ions et le rendement énergétique (cf. eqs (1) et (3)).

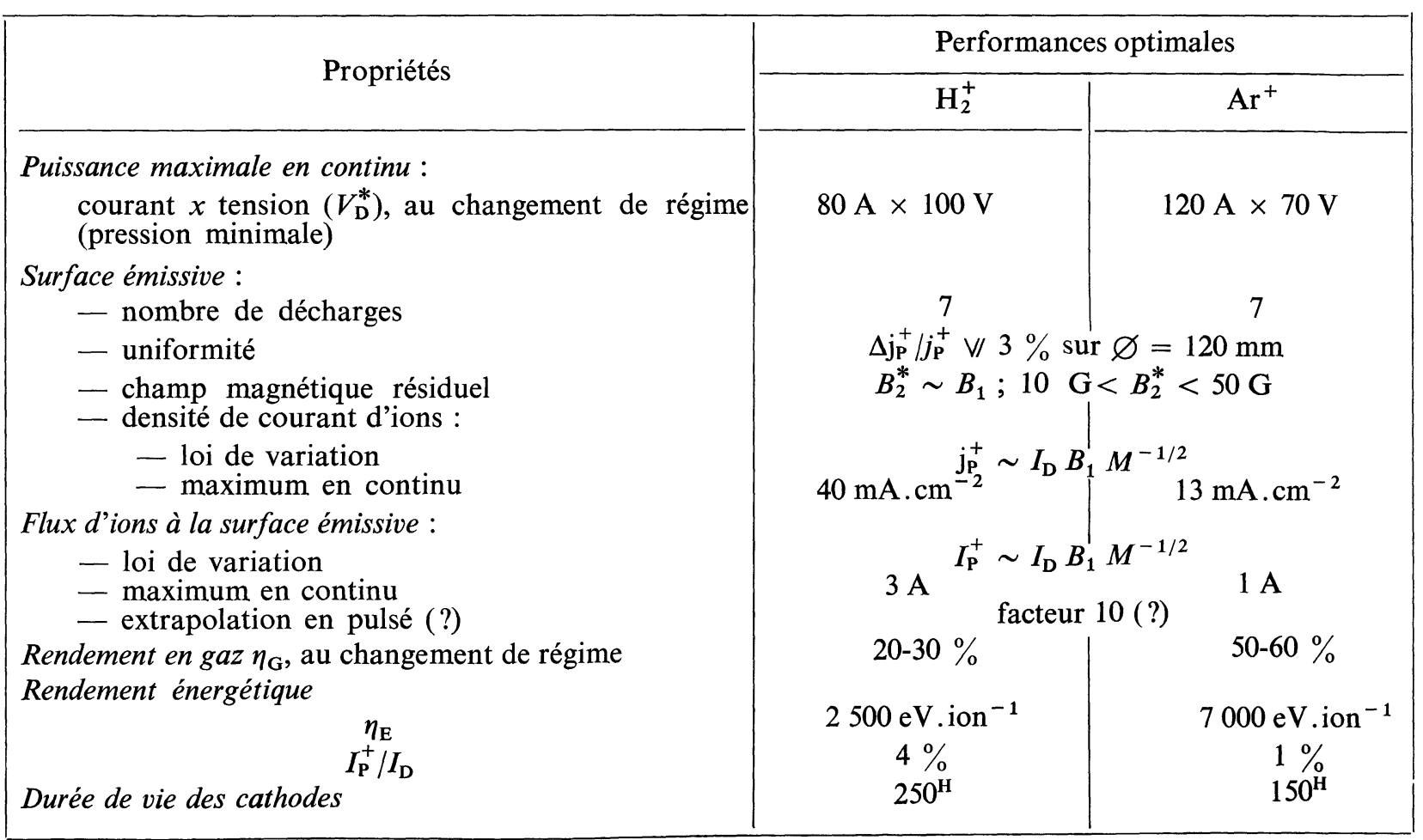


optimisée, la qualité du profil de densité n'est pas altérée par une variation des courants de décharge, qui conserve entre eux les proportions optimales; on a :

$$
I_{\mathrm{P}}^{+} \sim I_{\mathrm{D}} \text { et } j_{\mathrm{P}}^{+} \sim I_{\mathrm{D}} \text {. }
$$

- Les relations (4) et (5) ne sont satisfaites que dans la mesure où le régime d'appauvrissement en neutres des décharges n'est pas atteint, ce qui suppose une pression de neutres (ou un débit) supérieure à sa valeur minimale, fonction croissante de $B_{1}$ et $I_{\mathrm{D}}$. Ces derniers paramètres étant fixés, une augmentation de pression dans la décharge n'entraîne qu'un faible élargissement du profil de densité, en relation avec l'importance croissante du rôle des collisions au sein du plasma d'expansion. La pression n'affecte ni $I_{\mathrm{P}}^{+}$, ni $j_{\mathbf{P}}^{+}$mais elle détermine en partie le rendement d'ionisation (cf. § 2.2.1) d'où finalement la loi de variation des courants d'ions à la surface émissive :

$$
j_{\mathrm{P}}^{+} \sim I_{\mathrm{P}}^{+} \sim I_{\mathrm{D}} B_{1} M^{-1 / 2} .
$$

2.2.3 Performances optimales. - Quelle que soit la masse des ions, l'allumage des sept décharges du Multiduoplasmatron permet de créer un plasma d'expansion dont la surface émissive dans le plan de sortie de la structure est uniforme à mieux que $\pm 3 \%$ sur des dimensions (110-120 mm) grandes par rapport au diamètre de la chambre d'expansion $(155 \mathrm{~mm})$. Le choix des paramètres de fonctionnement qui optimisent cette surface émissive n'est pas critique, et l'obtention des performances optimales de cette structure de source ne pose pas de problèmes difficiles; elles sont résumées dans le tableau I pour une géométrie dont les dimensions sont celles de la figure 2 , aussi bien pour l'hydrogène que pour l'argon.

La plage de variation du rendement d'ionisation correspond à son augmentation avec le courant de décharge par suite du phénomène d'appauvrissement en neutres de la colonne ionisée; des valeurs plus élevées seraient obtenues pour une opération en pulsé avec des courants plus forts que ceux indiqués. Le point faible de cette structure est la valeur modeste du rendement énergétique, cependant supérieure à celle des sources sans champ magnétique [12], une amélioration d'un facteur deux peut être espérée par un simple élargissement des fentes d'éjection des plasmas, dont la largeur 0,4 $\mathrm{mm}$ a été imposée par le banc de pompage. L'avantage d'une structure multidécharges ayant été mis en évidence en ce qui concerne l'uniformité de la surface émissive, nous avons cherché à améliorer le rendement énergétique par la mise en œuvre de l'effet reflex sur des électrons.

3. Multiduopigatron. - 3.1 AdAPTATION DE LA STRUCTURE Multiplasmatron. - 3.1.1 Duopigatron: propriétés générales. - Le duoplasmatron à effet reflex (Fig. 9), introduit par Demirkhanov et son équipe [13], a été développé pour la formation de faisceaux de plus en plus intenses [14] ; il est désormais

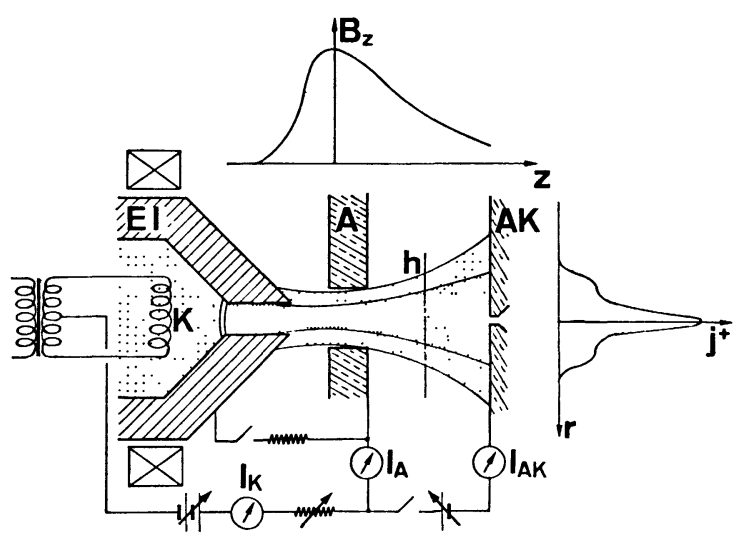

FIG. 9. - La structure Duopigatron ; schéma et circuit de polarisation de ses électrodes : $\mathrm{K}$ cathode chauffée ; $\mathrm{A}$ anode amagnétique ; EI électrode intermédiaire et $\mathrm{A}$. $\mathrm{K}$. anticathode. Les pôles de la lentille magnétique sont E. I. et A. K. ; $B_{z}=f(z)$ est le profil de l'induction magnétique le long de l'axe. Le graphe $j^{+}=f(r)$ donne une allure type de la variation du courant de saturation ionique d'une sonde cylindrique déplacée le long d'un diamètre dans un plan de section droite de la colonne de plasma magnétisé (ici h) : la présence d'un faisceau d'électrons primaires confinés au voisinage de l'axe y est mise en évidence.

[The duopigatron structure : $\mathrm{K}:$ heated cathode; $\mathrm{A}:$ anode ; E. I. : intermediate electrode ; A. K. : anticathode. The poles of the magnetic lens are $\mathrm{EI}$ and $\mathrm{AK}: B_{z}=f(z)$ is the axial magnetic field variation. The curve $j^{+}=f(r)$ gives a typical variation of the ionic saturation current of a probe with the position along a diameter of the plasma column (in the plane h).]

connu sous le nom de Duopigatron [15]. A la double compression - géométrique puis magnétique - de la décharge, est associé un piégeage partiel des électrons ionisants. Il est obtenu, axialement, par l'effet de potentiels adéquats appliqués à l'électrode intermédiaire EI et à l'anticathode $\mathrm{AK}$, et radialement par le confinement assuré par le champ magnétique axial. Ces processus, analogues à ceux mis en œuvre dans les décharges dites reflex ou $P I G$ [16] justifient le nom de la structure. Ils ont pour effet d'augmenter le temps de séjour des électrons injectés ou créés dans la colonne de plasma, ce qui améliore leur efficacité d'ionisation. Les mécanismes de création et transport des charges qui déterminent l'état d'équilibre et les propriétés d'une telle décharge ne sont pas encore bien établis. Une étude systématique en est faite dans notre laboratoire $[17,18]$. Nous rapportons sommairement et sans démonstration, une description de la dynamique de la décharge, afin de permettre au lecteur de mieux appréhender les adaptations nécessaires à l'optimisation du Multiduopigatron.

- La répartition longitudinale de potentiel est comparable à celle d'un duoplasmatron [9] ; selon la géométrie du canal de l'électrode intermédiaire et la topographie du champ magnétique dans ce canal, la double compression de la décharge donne naissance soit à 1 , soit à 2 gaines de charge d'espace séparant des plasmas quasi-équipotentiels. Il peut en résulter l'établissement de modes dont le comportement et les performances diffèrent notablement aussi bien quant 
au flux d'ions émis que pour le niveau général de bruit des plasmas. Un soin particulier doit donc être accordé à la réalisation de cette zone de compression de la décharge. Cependant, aucun critère d'application générale n'a pu être dégagé quant à l'influence des paramètres géométriques ; en particulier des différences de comportement de la double compression lorsque l'on passe de l'argon à l'hydrogène sont à noter (cf. Tableau II).

- Un faisceau d'électrons primaires est extrait du plasma occupant la région cathodique, puis accéléré par la d.d.p. qui s'établit dans la gaine de compression géométrique qui, pour obtenir un régime normal, doit se former à l'entrée du canal intermédiaire. Ce faisceau peut être mis en évidence - optiquement ou par sondes (Fig. 9) - tout le long de la colonne anodique et une maxwellianisation ultrarapide (paradoxe de Langmuir [19]) consécutive au développement d'instabilités du type faisceau-plasma est à écarter. L'enveloppe du faisceau est - en première approximation assimilable à un tube de force dont la forme dépend de la lentille magnétique et des conditions d'injection des électrons, c'est-à-dire de la géométrie du canal intermédiaire et du système de gaines qui s'y établit.

- Ce faisceau d'électrons primaires assure la majeure partie de l'ionisation de la colonne de plasma anodique ; il ne subit l'effet de piégeage dans la structure que s'il n'est pas intercepté par l'anode mais réfléchi par une anticathode. L'efficacité du piégeage radial est d'autant meilleure que : 1) le tube de force de confinement est, au niveau de l'anode, de dimensions petites par rapport à celles de cette anode et, 2) le temps de transit des électrons primaires entre le tube de force de confinement et l'anode est grand. Ce dernier dépend des processus responsables du transport radial des charges ; le développement d'instabilités, soit propres au plasma magnétisé, soit initiées par les oscillations des gaines de constriction, réduit fortement l'efficacité du piégeage des électrons [17].

- Le potentiel flottant de l'anticathode est intermédiaire entre les potentiels de l'anode et de la cathode. La polarisation négative de $\mathrm{AK}$, par rapport à son potentiel flottant, se traduit par une caractéristique de collection ionique présentant une saturation $\left(I_{\mathrm{AK}}^{+}\right)$ du flux d'ions, si le courant global $I_{\mathrm{A}}$ collecté par l'anode est maintenu constant ; ce dernier est considéré comme étant le courant de la décharge : $I_{\mathrm{D}} \equiv I_{\mathrm{A}}$. Dans ces conditions, la tension de décharge $V_{\mathrm{D}}$, celle entre anode et cathode, est réduite de 5 à $10 \%$ selon les neutres et le point de fonctionnement; un gain équivalent sur le rendement énergétique de production des ions en résulte, consécutif à l'amélioration du piégeage longitudinal des électrons primaires. La saturation ionique est déjà atteinte pour un potentiel égal à celui de la cathode ; par suite le contact électrique entre les deux électrodes $\mathrm{K}$ et $\mathrm{AK}$ assure une polarisation efficace de l'anticathode.

Le courant de saturation ionique $I_{\mathrm{AK}}^{+}$représente le flux d'ions disponible et pouvant être extrait axialement du duopigatron, équivalent au flux $I_{\mathrm{P}}^{+}$de la structure précédente. Le courant de faisceau sera :

$$
I_{\mathrm{F}}^{+}=t I_{\mathrm{AK}}^{+}
$$

$t$ étant la transparence de l'optique d'extraction dont la première électrode électrode écran est l'anticathode de la décharge $\left({ }^{1}\right)$. Les performances de la source de plasma sont caractérisées par le flux d'ions $I_{\mathrm{AK}}^{+}$, le rendement énergétique de production de ces ions :

$$
\eta_{\mathrm{E}}=I_{\mathrm{D}} V_{\mathrm{D}} / I_{\mathrm{AK}}^{+}
$$

et le rendement d'ionisation :

$$
\eta_{\mathrm{G}}=I_{\mathrm{F}}^{+} / e N_{0},
$$

$I_{\mathrm{F}}^{+}$étant mesuré dans notre expérience par collection sous basse tension $(2 \mathrm{kV})$. Le rapport $f=I_{\mathrm{AK}}^{+} / I_{\mathrm{D}}[10]$ peut également servir à caractériser le rendement énergétique ; il est appelé facteur d'illumination de l'anticathode.

\subsubsection{Multiduopigatron: Paramètres de construc-} tion. - L'optimisation du Multiduopigatron porte sur l'amélioration simultanée de $f$ et de l'uniformité de densité de la surface émissive du plasma. L'influence du champ magnétique y est primordiale car il joue un triple rôle : compression des plasmas au niveau des canaux intermédiaires, confinement des faisceaux d'électrons au niveau de l'anode et expansion des plasmas au niveau de l'anticathode au voisinage de laquelle le champ résiduel doit être aussi faible que possible. Aussi, les principales modifications ont eu pour objet l'adaptation au problème de la topographie du champ magnétique. L'évolution de la structure a été déterminée de façon méthodique sur la base des phénomènes rappelés et après analyse du comportement des décharges opérant soit séparément, soit simultanément. Nous avons adopté les solutions suivantes (Fig. 10) :

- L'anticathode amagnétique (AK) : le plan choisi pour positionner cette électrode est le plan de sortie de la chambre d'expansion; il n'est donc pas modifié par rapport au multiduoplasmatron. Ce choix se justifie par les mêmes raisons, auxquelles il faut ajouter la diminution de la pression minimale de fonctionnement de la décharge lorsque la longueur de la colonne augmente, ce qui entraîne une amélioration du rendement en gaz [18]. L'anticathode (en cuivre) est percée de trous circulaires $(\varnothing=4 \mathrm{~mm})$ répartis le long de deux diamètres perpendiculaires : il est ainsi possible

(1) Note : Pour le cas usuel des sources intenses, le plasma est suffisamment peu dense pour qu'en présence du champ extracteur il demeure contenu à l'intérieur de la décharge ; la transparence $t$ est alors déterminée essentiellement par les facteurs géométriques. Pour le cas de source avec expansion de plasma à partir d'un orifice de petites dimensions (cas de formation d'ions multichargés), l'épaisseur de la gaine de charge d'espace affecte la transparence de l'anticathode, qui dépend alors de sa polarisation. 


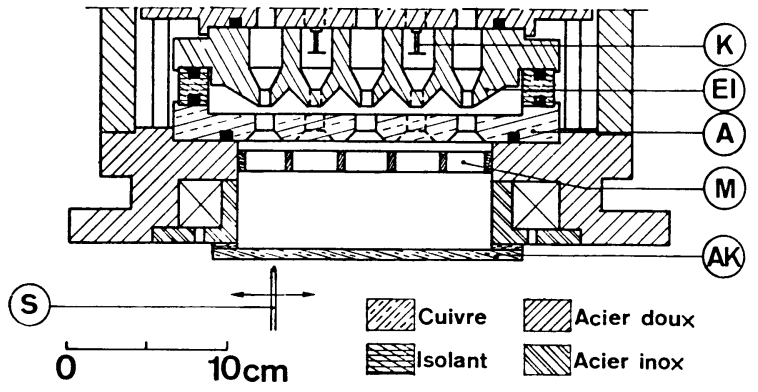

FIG. 10. - La structure Multiduopigatron optimisée : à comparer à celle du Multiduoplasmatron (Fig. 1) : K, quatre cathodes chauffées, celles des décharges médianes ; EI, électrode intermédiaire non modifiée ; $A$, anode amagnétique ; $M$, anticathode intermédiaire magnétique; $\mathrm{AK}$, anticathode amagnétique; $\mathrm{S}$, sonde électrostatique pour la mesure des profils de densité ionique. Le reste du circuit magnétique est inchangé.

[The optimized Multiduopigatron structure, to be compared to that of the Multiduoplasmatron (Fig. 1) : (1) four heated cathodes within the median discharge chambers ; (2) unmodified intermediate electrode ; (3) copper anode A ; (4) mild steel intermediate anticathode M ; (5) copper anticathode AK ; (6) Langmuir probe ; the other parts of the magnetic circuit are unchanged.]

d'explorer les propriétés du plasma effusant par ces orifices tout en limitant la conductance à l'électrode à une valeur compatible avec l'équipement de pompage. On peut supposer que cet artifice géométrique conduit cependant à l'obtention de résultats valables et extrapolables puisque les conditions aux limites ainsi imposées au plasma des décharges sont identiques à celles résultant de l'application d'un champ électrique extracteur pour les ions, au niveau d'une électrode écran percée de trous (ou de fentes) sur l'ensemble de sa surface. La sonde électrostatique se déplace quelques millimètres en retrait du plan de AK.

- L'anode amagnétique (A) : la suppression des plaques de molybdène qui obturent les fentes de l'anode en acier doux est insuffisante pour obtenir l'effet duopigatron: la trop forte divergence des tubes de force entraîne l'interception par l'anode de la majeure partie des faisceaux d'électrons primaires. Une pièce identique en cuivre a été adaptée.

- L'anticathode intermédiaire magnétique $(\mathrm{M})$ : l'anode amagnétique ne suffit pas à résoudre seule le problème de l'interception des faisceaux d'électrons primaires, dans le cas des décharges médianes et pour les valeurs croissantes du champ de compression (cf. $\S 3.2$ et Fig. 12). De plus, pour une telle géométrie, un profil de densité uniforme ne peut être obtenu que pour un champ $B_{2}$ de sens opposé à $B_{1}$. Le cusp ainsi crée favorise les pertes sur les parois et le facteur d'illumination est faible (Tableau II, ligne 1). Un deuxième pôle s'impose pour la lentille magnétique ; une électrode intermédiaire $M$ en acier doux percée de sept fentes sur le modèle de l'anode est introduite entre A et AK. Isolée, sa polarisation constitue un paramètre indépendant de fonctionnement. Les autres éléments du multiplasmatron sont inchangés et les principales adaptations des paramètres de construction du multiduopigatron ont porté successivement sur la position et l'épaisseur de $M$, la largeur des fentes d'anode et de $M$, et enfin celle des fentes de l'électrode intermédiaire.

\subsection{Résultats eXPérimentauX. - 3.2.1 Optimi-} sation des performances. - Quatre décharges duopigatron - symétriques en position médiane (Figs. 1 ou 10) - suffisent pour obtenir un large plateau de densité ionique. L'évolution des performances optimales en fonction des paramètres de construction est synthétisée dans le tableau II ; y figurent les valeurs des paramètres de fonctionnement conduisant à l'optimisation simultanée du facteur d'illumination, alors noté $f^{*}$, et de l'uniformité de densité de la surface émissive. La position aussi bien que les dimensions de $\mathbf{M}$ - épaisseur

\section{TABLEAU II}

Evolution des «performances optimales» du Multiduopigatron en fonction de la largeur des fentes dans les trois électrodes EI, A et M. Quatre décharges en position médiane (cf. Fig. 10) sont allumées $\left({ }^{*}\right)$. La largeur du plateau de densité est déterminée à partir du profil de densité le long d'un diamètre perpendiculaire au plan des décharges, en prenant pour critère une modulation de $\pm 2 \%$ de la densité.

\begin{tabular}{|c|c|c|c|c|c|c|}
\hline \multirow{4}{*}{ Gaz } & \multirow{4}{*}{$\begin{array}{c}\text { Paramètres } \\
\text { de construction : } \\
\text { largeur des fentes } \\
\text { (en } \mathrm{mm} \text { ) dans EI, A et } \mathrm{M}\end{array}$} & \multicolumn{5}{|c|}{ Performances optimales } \\
\hline & & \multicolumn{3}{|c|}{ Paramètres de fonctionnement } & \multicolumn{2}{|c|}{ Performances } \\
\hline & & & & & Largeur du & \\
\hline & & $B_{1}(\mathrm{~A})$ & $B_{2}(\mathrm{~A})$ & $V_{\mathrm{D}}^{*}(\mathrm{~V})$ & $\begin{array}{c}\text { densité à } \\
\pm 2 \%\left({ }^{*}\right)\end{array}$ & $f^{*}(\%)$ \\
\hline Argon & $\begin{array}{l}\text { EI }: 4 ; \mathrm{A}: 8 \text { (M absent) } \\
\text { EI }: 4 ; \mathrm{A}: 8 ; \mathrm{M}: 20 \\
\text { EI }: 4 ; \mathrm{A}: 12 ; \mathrm{M}: 20 \\
\mathrm{EI}: 4 ; \mathrm{A}: 12 ; \mathrm{M}: 26 \\
\mathrm{EI}: 6 ; \mathrm{A}: 12 ; \mathrm{M}: 26\end{array}$ & $\begin{array}{l}1 \\
1 \\
1 \\
0,8 \\
1\end{array}$ & $\begin{array}{l}-1 \\
+1 \\
+2 \\
+2 \\
+2\end{array}$ & $\begin{array}{l}50 \\
50 \\
60 \\
60 \\
60\end{array}$ & $\begin{array}{l}90 \mathrm{~mm} \\
80 \\
120 \\
120 \\
130\end{array}$ & $\begin{array}{r}3 \\
13 \\
23 \\
22 \\
20\end{array}$ \\
\hline Hydrogène & $\begin{array}{l}\mathrm{EI}: 4 ; \mathrm{A}: 8 ; \mathrm{M}: 20 \\
\mathrm{EI}: 4 ; \mathrm{A}: 12 ; \mathrm{M}: 26 \\
\mathrm{EI}: 6 ; \mathrm{A}: 12 ; \mathrm{M}: 26\end{array}$ & $\begin{array}{l}0,4 \\
0,4 \\
0,6\end{array}$ & $\begin{array}{l}+1,2 \\
+1,5 \\
+1,5\end{array}$ & $\begin{array}{r}80 \\
90 \\
115\end{array}$ & $\begin{array}{r}80 \\
110 \\
120\end{array}$ & $\begin{array}{l}18 \\
25 \\
42\end{array}$ \\
\hline
\end{tabular}


et largeur des fentes - établissent un compromis entre son action positive en tant qu'écran magnétique et son action négative en tant que collecteur d'ions et d'électrons ; une épaisseur de $12 \mathrm{~mm}$ pour une position proche de l'anode (Fig. 10) s'est avérée la plus favorable aussi bien pour $f^{*}$ que pour le plateau de densité. Pour des positions de $\mathbf{M}$ plus rapprochées de l'anticathode, des pics résolus apparaissent dans le profil de densité tant que $B_{1}$ et $B_{2}$ sont de même sens, condition nécessaire à l'obtention d'une valeur élevée pour $f^{*}$. Si les dimensions des fentes de $\mathbf{M}$ sont telles que les faisceaux d'électrons primaires ne sont pas interceptés, $f^{*}$ est peu sensible à la polarisation de $\mathbf{M}$ et cette électrode peut être laissée flottante. Pour une description plus complè̀e des performances, les conclusions suivantes, relatives à l'influence des paramètres de fonctionnement, sont à associer au tableau II.

- Influence du courant de décharge et de la pression des neutres. $-f^{*}$ est quasi-indépendant du courant de décharge dans la gamme explorée pour une opération en continu (0-25 A pour chaque décharge). Il en est de même pour l'uniformité du plasma. Par contre $f$ est une fonction décroissante de la pression des neutres dans la colonne anodique. En conséquence, pour que la propriété précédente soit satisfaite, il est nécessaire d'adapter le débit de gaz au courant de décharge. Le réglage optimal correspond, pour une géométrie et un champ magnétique donnés, à l'obtention d'une tension de décharge sensiblement constante, quel que soit $I_{\mathrm{D}}$ et dont la valeur $V_{\mathrm{D}}^{*}$, fonction des paramètres précéd $\epsilon$ nts, se situe entre 50 et $70 \mathrm{~V}$ pour l'argon et $100-150 \mathrm{~V}$ pour l'hydrogène. La décharge est alors au voisinage soit du changement vers le régime d'appauvrissement en

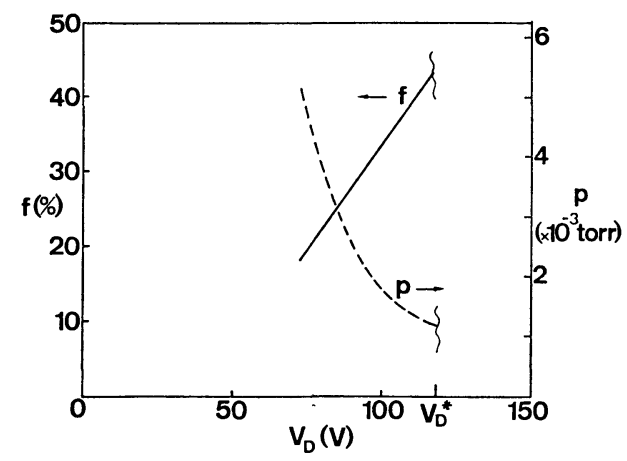

Fig. 11. - Multiduopigatron: facteur d'illumination $f$ de l'anticathode en fonction de la tension de décharge $V_{\mathrm{D}}$, dans l'hydrogène, à courant de décharge et champ magnétique constants : $I_{\mathrm{D}}=4 \times 8 \mathrm{~A} ; B_{1}: 0,6 \mathrm{~A} ; B_{2}: 1,5 \mathrm{~A}$. La pression doit alors être modifiée; sa variation est donnée sur l'échelle de droite. Au-delà de $V_{\mathbf{D}}^{*}=115 \mathrm{~V}:$ il y a extinction des décharges. La largeur des fentes des électrodes est respectivement : EI : $6 \mathrm{~mm}$; A : $12 \mathrm{~mm}$ et $\mathrm{M}: 26 \mathrm{~mm}$ (cf. Tableau II, dernière ligne).

[Multiduopigatron : variation of the illumination factor $f$ of the anticathode with the discharge voltage $V_{\mathrm{D}}$, the magnetic field strength and discharge currents being constant :

$I_{\mathrm{D}}=4 \times 8 \mathrm{~A} ; B_{1}: 0.6 \mathrm{~A} ; B_{2}=1.5 \mathrm{~A}$; gas : hydrogen.

The neutral pressure has to be adapted : its variation is referred to the righ-hand scale. Beyond $V_{\mathrm{D}}^{*}=115 \mathrm{~V}$ the discharges fall down. The width of the electrode slits are respectively :

$\mathrm{E}_{1}: 6 \mathrm{~mm} ; \mathrm{A}: 12 \mathrm{~mm}$ and $\mathrm{M}: 26 \mathrm{~mm}$ (cf. Table II, last line).] neutres, soit de l'extinction selon $B$ et la géométrie : $f$ atteint un maximum corrélé à une bonne stabilité de l'ensemble des plasmas de la décharge. La figure 11 illustre l'influence de la pression des neutres pour le cas particulièrement sensible de l'hydrogène. Pour la géométrie considérée ici, tableau II dernière ligne, le régime d'appauvrissement en neutres ne peut $\mathrm{p}$ a s'établir et l'on s'interrompt pour des valeurs de $V_{\mathbf{D}}^{*}$ $(\simeq 115 \mathrm{~V})$ insuffisantes pour assurer une valeur élevée du facteur d'illumination : une adaptation ultérieure de la géométrie s'impose. $V_{\mathrm{D}}^{*}$ est une fonction croissante de l'intensité du champ magnétique et de la largeur des fentes d'anode (cf. Tableau II) et son accroissement entraîne celui de $f^{*}$, d'où les conditions d'amélioration des performances de la source.

- Influence du champ magnétique. - $f^{*}$ ne correspond pas au maximum qu'il est possible d'atteindre par la variation du champ magnétique. La décroissance après passage par un maximum (Fig. 12) pour des



FIG. 12. - Multiduopigatron : facteur d'illumination en fonction de $B_{1}$ pour l'argon ; paramètre : le champ magnétique d'expansion $B_{2}$. Largeur des fentes dans les électrodes :A $12 \mathrm{~mm}$, EI $6 \mathrm{~mm}$, M $26 \mathrm{~mm} ; p_{\mathrm{A}}=5 \times 10^{-4}$ torr $; V_{\mathrm{D}}=60 \mathrm{~V} ; I_{\mathrm{D}}=4 \times 10 \mathrm{~A}$.

[Multiduopigatron : variation of $f$ with $B_{1}$ for the operation with Argon, the parameter being the expansion magnetic field $B_{2} ; V_{\mathrm{D}}=60 \mathrm{~V} ; I_{\mathrm{D}}=4 \times 10 \mathrm{~A} ; p_{\mathrm{A}} \simeq 5 \times 10^{-4}$ torr ; Electrode slits : EI : $6 \mathrm{~mm} ; \mathrm{A}: 12 \mathrm{~mm} ; \mathrm{M}: 26 \mathrm{~mm}$.]

valeurs de $B_{1}$ d'autant plus élevées que $B_{2}$ est intense, correspond à l'interception partielle par l'anode; la fraction interceptée est estimée d'après la répartition du courant de décharge entre $\mathrm{A}, \mathrm{M}$ et $\mathrm{AK}$, lorsque ces trois électrodes sont au même potentiel : l'effet reflex est supprimé et la décharge opère en duoplasmatron. L'élargissement des fentes de $\mathrm{A}$ et le positionnement adéquat de $M$ éliminent pratiquement ce phénomène en ce qui concerne la décharge centrale et les décharges médianes. Il n'en est pas de même pour les deux décharges extrêmes pour lesquelles, selon la position de $\mathbf{M}$, le maximum de $f$ est obtenu pour un champ $B_{1}$ trop faible (intensité magnétisante entre 0,3 et $0,5 \mathrm{~A}$ ) pour tirer le meilleur parti des performances des autres décharges. De plus, l'élargissement du plateau de densité qui résulte de l'allumage de ces deux décharges n'est pas significatif pour le plan d'anticathode choisi. 
Compte tenu des performances obtenues en se limitant à l'allumage des quatre décharges médianes, il ne nous a pas paru indispensable de rechercher une amélioration, soit par modification du circuit magnétique, soit par déplacement de l'anticathode. Les figures $13 a$ et $13 b$ montrent l'influence des champs $B_{1}$ et $B_{2}$ sur le profil transversal de densité ionique ; leur réglage, bien qu'important, n'est cependant pas critique pour l'obtention d'un plateau à faible modulation de densité. Comme pour le Multiduoplasmatron, la largeur importante des profils partiels de densité (Fig. 14) en sont la cause ; ici encore la superposition des décharges n'entraîne pas la sommation des densités partielles et le phénomène est plus accusé que dans le Multiduoplasmatron par suite de la superposition des milieux actifs des décharges, et non plus des jets de plasma qui en sont issus. Il est intéressant de noter que pour les géométries telles que le faisceau d'électrons primaires soit de dimension très inférieure à celle des fentes d'anode ( $A=12 \mathrm{~mm}$ ), les profils partiels présentent eux-mêmes un plateau (cf. Figs. 13 et 14) et non plus un profil en

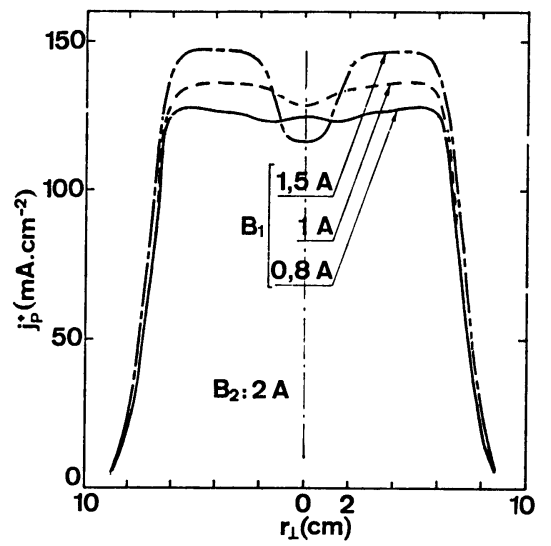

FIG. $13 a$



FIG. $13 b$

FIG. 13. - Multiduopigatron : influence du champ magnétique de compression $B_{1}(a)$ et d'expansion $B_{2}(b)$ sur le profil transversal de densité ionique; Argon ; largeur des fentes dans les électrodes : A $12 \mathrm{~mm}$, EI $4 \mathrm{~mm}, \mathrm{M} 26 \mathrm{~mm} ; p=5 \times 10^{-4}$ torr ;

$$
V_{\mathrm{D}}=60 \mathrm{~V} ; I_{\mathrm{D}}=4 \times 10 \mathrm{~A} \text {. }
$$

[Multiduopigatron : variation of the transversal profile of ion density with the compression (a) and expansion (b) magnetic fields; Argon $: p_{\mathrm{A}} \simeq 5 \times 10^{-4}$ torr $; V_{\mathrm{D}}=60 \mathrm{~V} ; I_{\mathrm{D}}=4 \times 10 \mathrm{~A}$; electrode slits : EI : $4 \mathrm{~mm}$; A : $12 \mathrm{~mm}$; $\mathrm{M}: 26 \mathrm{~mm}$.] REVUE DE PHYSIQUE APPLIQÚÉ. - T. 12, № 12, DÉCEMBRE 1977

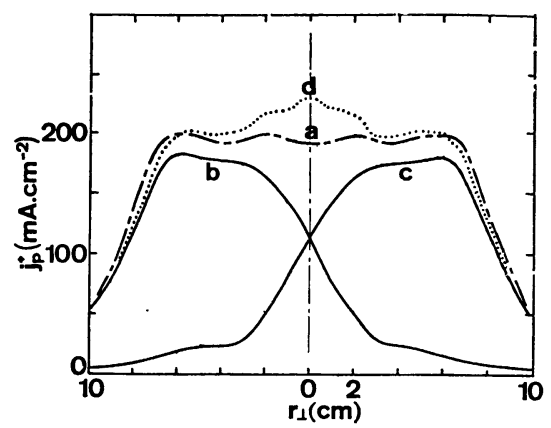

Fig. 14. - Multiduopigatron : superposition des plasmas dans le plan de l'anticathode: Gaz: Argon; $p=5 \times 10^{-4}$ torr ; $V_{\mathrm{D}}=60 \mathrm{~V} ; B_{1}: 1 \mathrm{~A} ; B_{2}: 2 \mathrm{~A}$; largeur des fentes : A : $12 \mathrm{~mm}$; $\mathrm{EI}: 6 \mathrm{~mm} ; \mathrm{M}: 26 \mathrm{~mm}$. a) profil réel pour quatre décharges $4 \times 18 \mathrm{~A}$; $b$ et $c$ ) profils partiels pour deux décharges $2 \times 18 \mathrm{~A}$; $d$ ) profil déterminé par sommation des profils $\mathrm{b}$ et $\mathrm{c}$.

[Multiduopigatron : plasma superposition at the anticathode plane ; Argon : $p_{\mathrm{A}} \simeq 5 \times 10^{-4}$ torr ; $V_{\mathrm{D}}=60 \mathrm{~V} ; B_{1}: 1 \mathrm{~A}$; $B_{2}: 2 \mathrm{~A}$; electrode slits : EI : $6 \mathrm{~mm}$; A : $12 \mathrm{~mm}$; $\mathrm{M}: 26 \mathrm{~mm}$. a) measured profile for 4 discharge operation: $4 \times 18 \mathrm{~A}$; b) and $c$ ) measured profile for 2 discharge operation : $2 \times 18 \mathrm{~A}$;

$d)$ calculated profile by addition of profiles $b$ and $c$.]

cloche. Cette observation, à rapprocher du profil obtenu pour un duopigatron cylindrique (Fig. 9) semble caractériser ce type de décharge reflex en champ magnétique fortement divergent; elle a déjà été mentionnée à propos des sources de propulseurs du type Kaufman [20].

3.2.2 Performances optimales. - Les meilleures performances du multiduopigatron sont résumées dans le tableau III. Pour cette structure, l'utilisation de quatre décharges identiques simplifie le réglage des courants ; compte tenu des deux plans de symétrie, ils doivent être égaux. De plus, pour une géométrie et des neutres donnés, l'intensité du champ magnétique optimum n'est pas un paramètre indépendant ; une construction à base d'aimants permanents peut être envisagée. On a donc finalement :

$$
I_{\mathrm{AK}}^{+}=f^{*} \text { (géométrie, neutre, pression) } \times I_{\mathrm{D}} .
$$

Pour le duopigatron, la puissance dissipée est mieux répartie entre les différentes électrodes que dans le duoplasmatron, pour lequel la majeure fraction est dissipée sur une faible zone de l'anode, ce qui entraîne des risques de destruction. Cependant, pour le prototype que nous avons étudié, en relation avec les modifications nécessaires à l'optimisation du rôle de $\mathbf{M}$, le refroidissement est insuffisant pour permettre un fonctionnement permanent et dans de bonnes conditions aux puissances supérieures à $4 \mathrm{~kW}$. L'échauffement de $M$ altère ses propriétés magnétiques avec pour conséquence la détérioration de l'uniformité de la surface émissive. Un refroidissement efficace de $M$ et $A K$ - comme l'est déjà celui de EI et A - devrait permettre le fonctionnement en continu du Multiduopigatron jusqu'à des puissances de l'ordre de $15 \mathrm{~kW}$. Ce travail d'un intérêt pratique n'a pas été entrepris, 


\section{TAbleau III}

Performances optimales du Multiduopigatron; (*) un refroidissement des anticathodes $M$ et AK, plus efficace que celui mis en auvre pour l'adaptation de ce prototype doit permettre de gagner un facteur 3 sur la puissance de fonctionnement en régime permanent; il en résulterait un gain équivalent sur le flux d'ions $\left(\mathrm{I}_{\mathrm{AK}}^{+}\right.$et $\left.j_{\mathrm{P}}^{+}\right)$. Pour l'hydrogène la largeur des fentes dans les électrodes (cf. Tableau II) sont : $E I: 6 \mathrm{~mm}, A: 12 \mathrm{~mm} ; M: 26 \mathrm{~mm}$; pour l'argon : $E I: 4 \mathrm{~mm} ; A: 12 \mathrm{~mm}$ et $M: 20 \mathrm{~mm}$.

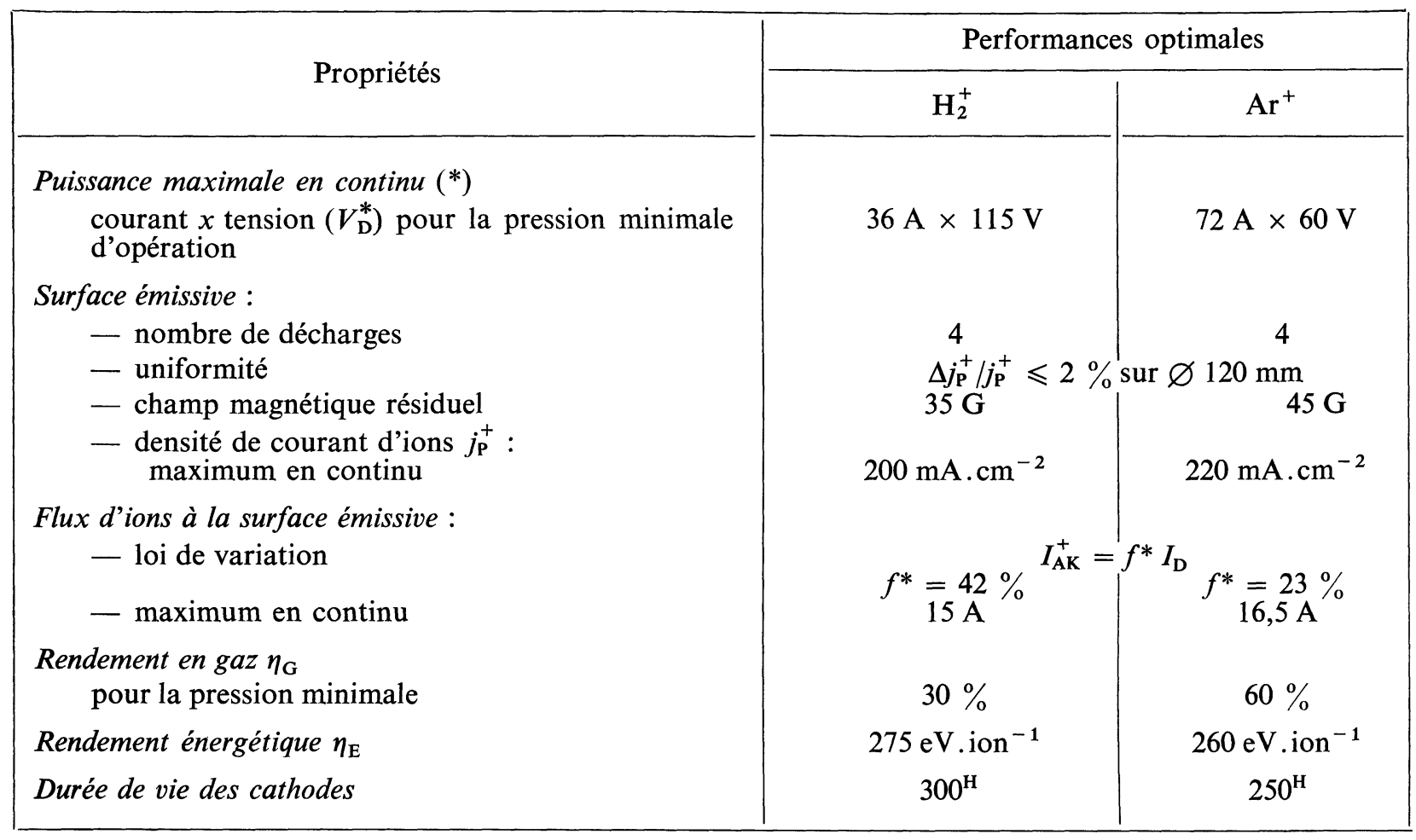

une source à surface émissive rectangulaire étant mieux adaptée aux applications actuelles des faisceaux d'ions intenses.

Une nouvelle amélioration de $f^{*}$ n'a pas été recherchée pour le prototype actuel opérant avec l'hydrogène. Elle semble possible, des valeurs de 50 à $55 \%$ étant obtenues par ailleurs pour $V_{\mathrm{D}}^{*}$ entre 130 et $150 \mathrm{~V}$. Un élargissement des fentes de l'électrode intermédiaire de 6 à $8 \mathrm{~mm}$ et de l'anode de 12 à $15 \mathrm{~mm}$ pourrait être envisagé ; Becherer [21] rapporte un accroissement de $f$ jusqu'à $50 \%$ par l'adaptation du canal intermédiaire. Pour l'argon $f^{*}=22 \%$ semble correspondre au compromis idéal entre la valeur maximale atteinte (30\%) et l'uniformité du plasma émissif. Pour les autres gaz les valeurs de $f^{*}$ sont comprises entre celles de l'hydrogène et l'argon, mais elles ne suivent pas la loi en $M^{-1 / 2}$, rencontrée pour le duoplasmatron.

En ce qui concerne les instabilités du plasma, pour les valeurs des champs magnétiques permettant une bonne uniformité spatiale, la fluctuation de la densité de courant ionique au niveau de la surface émissive est inférieure à $\pm 5 \%$, sur l'ensemble de cette surface. Par ailleurs, la structure discontinue de l'effusion du plasma depuis les trous de l'anticathode, n'apparaît plus sur les profils de densité de courant ionique dès que le plan de mesure par la sonde s'écarte de $5 \mathrm{~mm}$ du plan d'émission. Cette observation suppose une isotropie partielle de la fonction de distribution des vitesses ioniques au niveau de la surface émissive.

4. Discussion. - La comparaison des tableaux I et III montre que l'optimisation des décharges reflex du Multiduopigatron conduit, par rapport au Multiduoplasmatron, à un accroissement important de la densité du courant d'ions disponible à l'interface plasmafaisceau et, par suite, du flux d'ions total, tout en préservant une très bonne uniformité spatiale (modulation inférieure à $\pm 2 \%$ sur $120 \mathrm{~mm}$ de section) et un faible niveau de fluctuation temporelle (inférieur à $\pm 5 \%$ sur un grand nombre de périodes des oscillations). La confrontation avec les performances des sources d'ions légers positifs développées dans différents laboratoires - cf. la revue récente de Green [22] - nous permet de conclure qu'une telle structure réalise une synthèse des meilleurs résultats : le rendement énergétique des sources à décharge reflex Kaufman [20] ou Duopigatron $[5,21]$ et l'uniformité de densité des sources sans champ magnétique [12] ; pour une uni- 
formité comparable, le rendement énergétique est supérieur à celui du Périplasmatron [23], dont le principe - intermédiaire entre les deux précédents conduit à un premier compromis. Considérant, de plus, sa durée de vie - celle des cathodes à oxydes, soit 200-300 H - et la relative facilité de sa mise en œuvre et de son exploitation - quatre décharges de même intensité suffisant à assurer l'uniformité pour une chambre de $155 \mathrm{~mm}$ - le Multiduopigatron apparaît comme une alternative valable aux structures de décharge des sources intenses étudiées ou utilisées actuellement : elle fournit un plasma faiblement magnétisé, dense, stable, quasi-uniforme sur une surface de grande étendue, conditions simultanément nécessaires à la formation de faisceaux multiampères de faible divergence.

Pour être complet, il conviendrait de vérifier que ces conditions sont effectivement suffisantes. Ce n'est pas possible en régime pulsé avec notre banc d'essai, aussi bien pour des problèmes d'équipement de vide que d'alimentation de puissance, et la formation en continu de faisceaux multiampères pose des problèmes de tenue en tension et en température qui sont étudiés actuellement par différentes équipes. Ces expériences sortent du cadre des objectifs de développement des recherches de notre groupe universitaire. On peut cependant dire que pour le Multiduopigatron le plasma émissif est comparable à celui des Duopigatrons usuels, pour lesquels la détérioration des qualités optiques du faisceau a pu être reliée en premier lieu à la nonuniformité de la surface émissive [5]. Le plasma est celui d'une décharge faiblement magnétisée, régie par un modèle de chute libre pour les ions [24], les anisotropies de la fonction de distribution des vitesses des ions et des électrons sont donc réduites, en particulier si on les compare à celles mises en évidence pour le plasma d'expansion du Multiduoplasmatron - du type sans collisions. Des expériences conduites sur des sources de moindre intensité sont actuellement en cours afin d'analyser les émittances des faisceaux extraits de ces deux types de plasmas et d'établir des corrélations émittance-plasma émissif.

Nous avons dégagé, au cours de cette étude, les conditions d'optimisation d'un prototype pour l'illumination d'une surface circulaire, aussi bien pour des ions légers que des ions lourds. Les améliorations successives des performances - en particulier consécutives à l'élargissement des fentes d'anode dans le cas de l'argon, puis celui des fentes de l'électrode intermédiaire pour l'hydrogène - confirment le bien-fondé des mécanismes que nous avons présentés comme étant responsables du comportement et des propriétés du duopigatron; leur validité peut être mise à profit, dans un contexte plus général, pour la recherche de l'amélioration des performances des sources intenses qui utilisent cette décharge. Les résultats sont applicables à l'illumination d'une surface rectangulaire à partir de décharges linéaires, toutes identiques ; un ensemble de grandes dimensions à structure modulaire peut être imaginé comme une assemblée de modules élémentaires. Il est intéressant de noter que, pour une telle réalisation, un système de bobines d'excitation $B_{1}$ et $B_{2}$ autour de chaque module rectangulaire permettrait de résoudre les problèmes de retour de flux et donc de périodicité du champ magnétique, par l'inversion du sens du champ dans les cellules contigues. En ce qui concerne les applications au traitement des surfaces, il est important de noter que la structure Multiduopigatron semble bien adaptée à la production de faisceaux intenses d'ions métalliques, les neutres étant introduits par la pulvérisation, sous l'impact des ions, de chemises métalliques portées par l'électrode $\mathbf{M}$, dont le potentiel peut être porté jusqu'à 1 ou $2 \mathrm{kV}$ sans modifier le comportement de la décharge. Ce principe a déjà été exploité avec succès pour la production d'ions métalliques moyennement multichargés à partir d'un duopigatron [17].

Quant au Multiduoplasmatron, ses performances se situent au niveau des sources sans champ magnétique et donc peuvent limiter son domaine d'application pour la production d'ions positifs. Cependant cette structure paraît intéressante pour l'extraction directe de faisceaux intenses d'ions légers négatifs $\left(\mathrm{H}^{-}, \mathrm{D}^{-} \ldots\right)$ en vue de la génération future d'injecteurs de neutres, et c'est pourquoi il nous a paru utile de présenter ses propriétés. En effet, il est désormais classique d'extraire des ions négatifs de duoplasmatrons [25] soit à émission décentrée par rapport à l'axe (ou plan de symétrie) de la décharge, soit fonctionnant en décharge creuse ; ces adaptations sont réalisables avec le Multiduoplasmatron, l'introduction de Césium dans la cuvette d'expansion pouvant de plus être envisagée pour améliorer le rendement de production des ions négatifs.

Remerciements. - Les auteurs remercient le Professeur G. Gautherin pour les encouragements qu'il leur a prodigués et pour les discussions fructueuses au cours de ce travail. Ils remercient C. Mardirossian pour son assistance technique dans la réalisation du programme expérimental.

\section{Bibliographie}

[1] Morgan, O. B., Proc. 2nd Symposium on Ion Sources and Formation of Ion Beams. Berkeley 1974. Rep. LBL 3399 Papier VI. 1.

[2] Rose, P. H., Ib. ref. [1] Papier VII.1.

[3] Osher, J. E., Proc. 2nd Int. Conf. on Ion Sources. (Vienne) 1972 p. 234.
[4] Coupland, J. R., Green, T. S., Hammond, D. P. et Rivière, A. C., Rev. Sci. Instrum. 44 (1973) 1258.

[5] Davis, R. C., Jernigan, T. C., Morgan, O. B., Stewart, L. D. et Stirling, W. L., Rev. Sci. Instrum. 46 (1975) 576.

[6] Thompson, E., Ib. ref. [1] Papier II.7. 
[7] Osher, J. E., I. E. E. E. Trans. Nucl. Sci. 22 (1975) 1626.

[8] Lejeune, C., Prangère, F. et Septier, A., J. Physique Colloq. 29 (1968) C 3-86.

[9] Lejeune, C., Nucl. Inst. and Meth. 116 (1974) 417 ; Nucl. Inst. and Meth. 116 (1974) 429.

[10] Septier, A., Prangère, F., Ismail, H. et Gautherin, G., Nucl. Inst. and Meth. 38 (1966) 41.

[11] Delcroix, J. L., (Monographie Dunod) Physique des Plasmas 2 (1966) 92 ou Proc. 1 ${ }^{\text {re }}$ Conf. Int. Sources d'Ions. (Saclay) (1969) p. 1.

[12] Ehlers, R. W., Baker, W. R., Berkner, K. H., CoOper, W. S., Kunkel, W. B., Pyle, R. V. et Stearns, J. W., Ibid Ref. [1] papier I.5.

[13] Demirkhanov, R. A., Fröhlich, H., Kursanov, V. V., et GutKIN, T. I., " Collection of High Energy Accelerator Papers from U. S. S. R. » B. N. L. Report $\mathrm{n}^{\circ} 767$ (1962) 224.

[14] Fasolo, J., Proc. Proton Linear Accelerator Conf. Brookhaven (1968) BNL-50120, p. 306.

[15] Davis, R. C., Morgan, O. B., Stewart, L. D. et Stirling, W. L., Rev. Sci. Instrum. 43 (1972) 278.
[16] Green, T. S., Rep. Prog. Phys. 37 (1974) 1257-1344.

[17] Lejeune, C., Grandchamp, J. P., Gilles, J. P. et Aubert, J., I. E. E. E. Trans. Nucl. Sci. N. S.-23 (1976) 1084.

[18] Grandchamp, J. P., et Lejeune, C., Comm. au Congrès National de Physique des Plasmas. Paris Décembre 1976.

[19] Crawford, F. W. et Self, S. A., Int. J. Electr. 18 (1965) 569.

[20] KNAUER, W., Proc. 1st Symposium on Ion Sources and Formation of Ion Beams BNL Report 50310 (1971) p. 69.

[21] BeChERER, R., Rapport EUR-CEA-FC 788. Sept. 1975.

[22] Green, T. S., Conférence invitée présentée au Congrès National de Physique des Plasmas. Paris Déc. 1976.

[23] Fumeldi, M. et Valckx, F. P. G., Nucl. Instrum. and Meth. 135 (1976) 203 et Rapport EUR-CEA-FC-809 (1976).

[24] Self, S. A., Phys. Fluids 6 (1963) 1762.

[25] Prelec, K. et Sluyters, Th., Rev. Sci. Instrum. 44 (1973) 1451 . 